\title{
Design and Application of Large Torque Electrical Load Simulator for Positive and Reverse Operation
}

\author{
Zhiqiang Wei, Guanghua Zong, Guochen Niu, Xiaolei Zhang \\ Robotics Institute \\ Beihang University \\ Beijing, China \\ wzq761229@sohu.com
}

\begin{abstract}
In order to meet the testing requirement of positive and reverse operation large torque load for new rudder, an electrical load simulator is designed. The realization principle of system is given according to the system index, and the stability and so on. The system mathematical model is established and the feedforward compensation method of torque and rudder angle is adopted to restrain the surplus torque according to the principle of invariance. So the high precision large torque load under positive and reverse operation for rudder is realized by torque and position hybrid control. The practical application shows that the proposed method can effectively restrain the surplus torque and the system meets the high precision torque load under positive and reverse operation for rudder very well.
\end{abstract}

Keywords-electrical load simulator; positive and reverse operation; hybrid control ; feedforward compensation

\section{INTRODUCTION}

In order to meet the testing requirement of positive and reverse operation for new rudders, a stable and reliable torque loading system must be designed to realize the high precision load torque. Nowadays, there are few reports about the large electrical torque load under the reverse operation for rudder at home. So the electrical load system under the positive and reverse operation for rudder is firstly designed according to the practical need, which provides a technical support to improve performance and evaluate effectively for new rudders.

To restrain the surplus torque, the cascaded loops torque and position hybrid control is proposed on the basis of analyzing the system construction and the control method. The electrical load system with the high precision, high symmetry and high linearity is realized combining with rudder angle and torque feedforward control. Practical experiments show the effectiveness of the proposed approach.

\section{SYSTEM STRUCTURE}

Large torque motor is introduced in the reverse operation torque load system for rudder,it can makes full use of the merits of the motor such as large work torque, small torque ripple, and working in the stalling state for long term and so on. The system consists of torque motor with encoder, and coupling with high stiffness and high flexibility, torque sensor, spring stick, high precision encoder, rudder, power source, bearing and so on and it is shown in Fig.1.

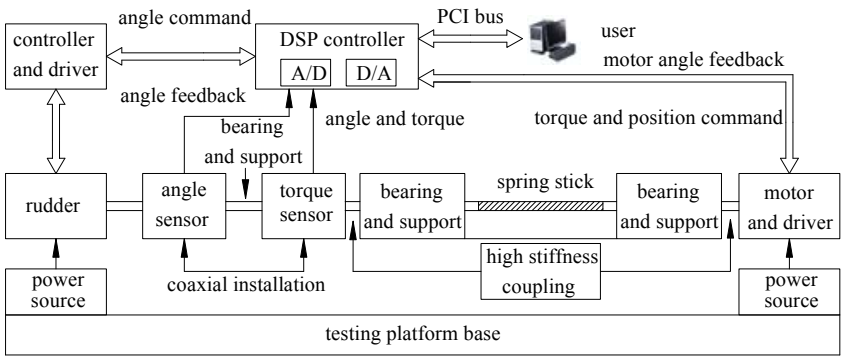

Figure.1 Electrical torque system of reverse operation torque load

Firstly, we make supplement for the definition of reverse operation in[1]: during the rudder working process, the reverse operation is the case that the direction of air load to the rudder is in accordance with the moving direction of the rudder, at this moment the rudder axis will deviate from the equilibrium position under certain air load conditions and the deflection angle will increase continuously. On the contrary, the positive operation is the case that the direction of air load to the rudder is contrary with the moving direction of the rudder, and at the same time the rudder axis will return to the equilibrium position under certain air load conditions accompanying the rudder deflection angle decreasing to zero.

Torque value is detected by high precision torque sensor and is sent into motion controller to form a closed loop torque control. The rudder rotation angle variation is measured by the high precision encoder, which together with the converted torque signal by $\mathrm{A} / \mathrm{D}$ converter are stored into the controller. Thus actual torque for reverse operation imposed on the rudder will be obtained. The spring stick as the torque buffer in the system can suppress high frequency disturbance caused by the rudder position variation, which can improve the performance of the loading system.

\section{System MAthematic Model}

\section{A. System Model Analysis}

To analyze the model conveniently, some assumptions are made as below[2]: 
1) The whole axis structure is connected by rigid coupling except the spring stick;

2) Large torque motor is adopted in the system, and its moment of inertia is relative large, so the moment of inertia of the shaft is transformed equivalently into that of the motor shaft.

\section{B. Model of Motor and Drive}

Voltage balance equation and the torque balance equation of motor are described as below[3-4]:

$$
\left\{\begin{array}{l}
U_{m}=i_{m} R_{m}+L_{m} \frac{d i_{m}}{d t}+K_{E} \frac{d \theta_{m}}{d t} \\
T_{m}=J_{m} \frac{d^{2} \theta_{m}}{d t}+B_{m} \frac{d \theta_{m}}{d t}+T_{L}
\end{array}\right.
$$

Where, $U_{m}$ is the voltage of the armature, $i_{m}$ is the current of the armature, $R_{m}$ is the equivalent resistance of the motor, $L_{m}$ is the equivalent inductance of the motor, $K_{E}$ is the back EMF constant of the motor, $\theta_{\mathrm{m}}$ is the rotary angle of torque motor, $T_{m}$ is the motor output torque, $J_{m}$ is the moment of inertia of motor and load, $B_{m}$ is the damping coefficient, $T_{L}$ is the load torque.

The model of motor drive can be described as below:

$$
K_{p}=\frac{U_{m}}{u_{i}}
$$

Where $u_{i}$ is the input signal.

\section{Model of Spring Stick}

Ignoring moment of inertia of the spring stick and the torque transmission loss, the coupling with high stiffness and the torque sensor is installed between the torque motor and the top of the spring stick, whose equivalent stiffness is much higher than the stiffness of the spring stick. So, the torque motor and spring stick are regarded as rigid connection. Similarly, the connection between the end of the spring stick and the rudder axis is also rigid,. The relationship between the angle variation of the end of the spring stick and the torque imposed on the rudder $T_{L}$ can be described as below:

$$
T_{L}=K_{L}\left(\theta_{m}-\theta_{a}\right)
$$

Where, $K_{L}$ is the stiffness coefficient of the spring stick, $\theta_{m}$ is the rotary angle of the motor, $\theta_{a}$ is the rotary angle of rudder.

Based on the above three parts, the dynamic model are established as a two-mass-spring system. Meanwhile, the objective is to trace the torque instruction accurately, which has no direct relationship with the rudder model, and the command signal of the rudder and the rudder angular displacement are both the physical quantities which can produce the surplus torque[3]. So ignoring the factor of rudder model, the transfer function of the motor load torque related with the control input and the rudder rotary angle can be described as below:

$$
T_{L}(s)=\frac{A(s) u_{i}(s)-B(s)}{C(s)}
$$

Where,

$$
\begin{aligned}
& A(s)=K_{p} K_{T} K_{L} \\
& B(s)=K_{L}\left[J_{m} L_{m} s^{2}+\left(J_{m} R_{m}+B_{m} L_{m}\right) s+\left(B_{m} R_{m}+K_{E} K_{T}\right)\right] s \theta_{a}(s) \\
& C(s)=J_{m} L_{m} s^{3}+\left(J_{m} R_{m}+B_{m} L_{m}\right) s^{2}+\left(B_{m} R_{m}+K_{E} K_{T}+K_{L} L_{m}\right) s+K_{L} R_{m}
\end{aligned}
$$

\section{Determination OF SPRING Stick StiffNeSS}

If the stiffness coefficient is small, the dynamic response of the system is slow and the bandwidth is narrow. Otherwise the system stability will decline, which increases the difficulties in system control and eliminating the surplus torque[5]. In order to guarantee the system stability and control capability and also meet the requirement of the loading frequency band, we assume that the system works under the input of sinusoidal signal and determine the range of the spring stick stiffness coefficient based on the following constraint conditions.

Supposing that the rudder shaft is fixed and static, the torque input can be described as below:

$$
\text { Torque }(t)=T \sin (2 \pi f t)
$$

Where, $f$ is the imposed frequency of sinusoidal torque signal and $T$ is the amplitude. Meanwhile, supposing that the motion equation of the motor is as below:

$$
\theta_{m}(t)=A_{m} \sin (2 \pi f t)
$$

Where $A_{\mathrm{m}}$ is motion amplitude of the motor:

$$
A_{m}=\frac{T}{K_{L}}
$$

The angular velocity and the angular acceleration of the torque motor can be obtained from (5).

$$
\left\{\begin{array}{l}
\omega_{m}(t)=2 \pi f A_{m} \cos (2 \pi f t) \\
\alpha_{m}(t)=-4 \pi^{2} f^{2} A_{m} \sin (2 \pi f t)
\end{array}\right.
$$

The angular velocity and the angular acceleration of the motor are always within the rated angular velocity and the maximum acceleration capacity. The following expression can be derived from (6), (7) and (8):

$$
\left\{\begin{array}{l}
K_{L}>\frac{2 \pi f T}{\omega_{\max }} \\
K_{L}>\frac{4 \pi^{2} f^{2} T}{\alpha_{\max }}
\end{array}\right.
$$

We can learn from (9) that there is a minimum value of the spring stick stiffness.Moreover,to avoid mechanical resonance, the system intrinsic frequency should be improved to be outside of the bandwidth of the system. So the bandwidth $f_{H}$ can be described as below:

$$
\sqrt{\frac{K_{L}}{4 \pi^{2} J_{m z}}}>f_{H}
$$

Supposing that motor works in the low-frequency condition, at this time we could ignore the motor torque loss caused by the damping and the maximum motor angular acceleration when the motor is detached with the rudder shaft can be described as below: 


$$
T_{\max }=J_{m z} \alpha_{\max }
$$

Where $T_{\max }$ is the maximum torque of the motor, $J_{m z}$ is the total moment of inertia including the moment of inertia of motor and the equivalent load moment of inertia converted to the motor shaft. Combining (9) and (10), and according to the parameters in TABLE I and in view of the different load torque range of the different rudder, we choose the spring stick stiffness $K_{L}$ to be $2091 \mathrm{Nm} / \mathrm{rad}$ and $4184 \mathrm{Nm} / \mathrm{rad}$, and we can choose different spring sticks to obtain the best loading performance for different load torque.

TABLE I. SYSTEM PARAMETERS

\begin{tabular}{|c|c|c|c|}
\hline parameter & value & parameter & value \\
\hline$f_{H} / \mathrm{Hz}$ & 25 & $\omega_{\max } /(\mathrm{rad} / \mathrm{s})$ & 20.94 \\
\hline$T_{\max } /(\mathrm{Nm})$ & 500 & $\alpha_{\max } /\left(\mathrm{rad} / \mathrm{s}^{2}\right)$ & 1552.80 \\
\hline
\end{tabular}

\section{CONTROller Design}

Based on the system mathematic model, the whole control system is shown in Fig.2.Cascaded loops torque and position hybrid control mode is introduced, in which the outside loop is the torque loop while the inside three loops consists of the current loop, the velocity loop and the position loop in turn from within.

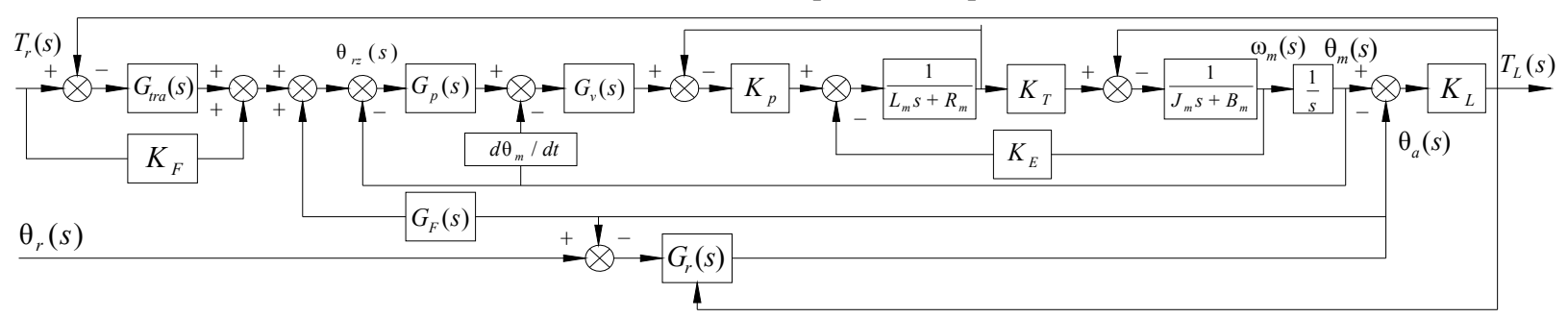

Figure.2 The whole structure of control system

$G_{r}(s)$ and $\theta_{r}(s)$ are respectively the transfer function and the position command, $G_{\text {tra }}(S)$ and $K_{F}$ are respectively the torque controller and the feedforward factor. The flexibility segment of spring stick increases the system stability, but it also has a bad influence on the system response speed and the control precision. The torque feedforward can improve the response speed.In addition, the active motion of the rudder will produce position disturbance. So according to principle of invariance, the system with two degrees of freedom is established after introducing torque feedforward control and rudder angle compensation[6].The structure diagram of principle of invariance is shown in Fig.3[7].

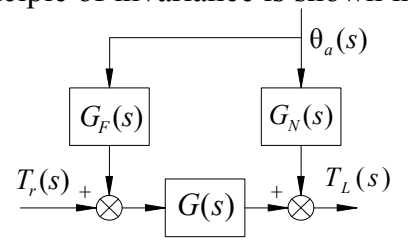

Figure.3 The structure chart of principle of invariance

It can be seen that the $G_{F}(s)$ should meet $G_{N}(s)=G_{F}(s)$ $G(s)$ so as to eliminate the disturbance caused by the variation of the rudder angle. According to the structure diagram, transfer function $G(s)$ from command torque to the output torque and $G_{N}(s)$ from rudder angle to the output torque are as below:

$$
\left\{\begin{array}{l}
G(s)=\frac{T_{L}(s)}{T_{r}(s)}=\frac{N(s)}{D(s)} \\
G_{N}(s)=\frac{T_{L}(s)}{\theta_{a}(s)}=\frac{N_{N}(s)}{D(s)}
\end{array}\right.
$$

Where,

$N(s)=\left(K_{F}+G_{t r a}(s)\right) G_{p}(s) G_{v}(s) K_{p} K_{T} K_{L}$
$N_{N}(s)=K_{L}\left[J_{m} L_{m} s^{3}+\left(J_{m} R_{m}+B_{m} L_{m}\right) s^{2}+\right.$

$\left.\left(B_{m} R_{m}+K_{E} K_{T}+K_{L} L_{m}\right) s+K_{p} K_{T} G_{p}(s) G_{v}(s)\right]$

$D(s)=J_{m} L_{n} s^{3}+\left(J_{m} R_{m}+B_{m} L_{m}\right) s^{2}+\left(B_{m} R_{m}+K_{E} K_{T}+K_{L} L_{m}\right) s+$

$K_{L} R_{m}+K_{p} K_{T} K_{L} G_{v}(s)+K_{p} K_{T} K_{L} G_{p}(s) G_{v}(s)+K_{p} K_{T} K_{L} G_{r r a} G_{p}(s) G_{v}(s)$

$G_{F}(s)=\frac{J_{m} L_{m}}{G_{p}(s) G_{v}(s) K_{p} K_{T}} s^{3}+\frac{J_{m} R_{m}+B_{m} L_{m}}{G_{p}(s) G_{v}(s) K_{p} K_{T}} s^{2}+$

$\frac{B_{m} R_{m}+K_{E} K_{T}+K_{L} L_{m}}{G_{p}(s) G_{v}(s) K_{p} K_{T}} s+1$

If the position loop and velocity loop are respectively based on the proportional control and the proportional integral control, the four items in $G_{F}(s)$ correspond respectively to the compensation segment to disturbance torque from rudder acceleration variation, angular acceleration, angular velocity and the angle. There is a highorder differential coefficient in each item 1,2,3 in (13), which is difficult to realize in engineering. So only the item 4,i.e., the compensation of rudder angle is introduced.

\section{EXPERIMENT AND ANALYSIS}

Maximum load capacity of the test equipment is $500 \mathrm{Nm}$. During the testing experiment of the equipment maximum capacity, the S-curve acceleration and deceleration control algorithm is adopted based on position loop so as to smooth the load torque of the torque closed loop, which can avoid the damage to the equipment structure and the sensors because of the over loading. Fig.5 shows that the load torque increases from $0 \mathrm{Nm}$ to $500 \mathrm{Nm}$ at a $10 \mathrm{Nm}$ step and then decreases from $500 \mathrm{Nm}$ to $0 \mathrm{Nm}$ at the same step. It also shows that the maximum load torque of $500 \mathrm{Nm}$ can be accurately reached no matter in positive or negative 
direction. Moreover, PID parameters adaptive control is adopted based on a large number of experiments aiming at different load torque, so the good torque symmetry and system stability are guaranteed.

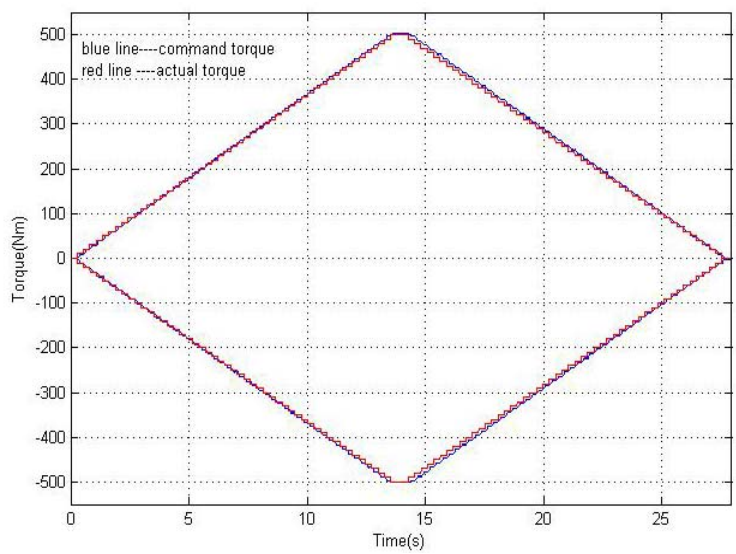

Figure.5 Testing chart of maximum loading capacity

According to the positive and reverse operation torque index of a certain rudder, practical experiment is performed. Fig. 6 shows the curve of reverse operation imposing $100 \mathrm{Nm}$ on the rudder.The left $\mathrm{Y}$ axis represents the position of loading motor and the rudder while the right $\mathrm{Y}$ axis represents the load torque.The process of reverse operation is as follows: the motor and rudder firstly return to zero, meanwhile, they move positively deviating the equilibrium position, and at about 0.7 second $100 \mathrm{Nm}$ torque is then impacted on the rudder because of the position difference between them, and then they move continuously with the torque, which finally produces $10^{\circ}$ rudder deviation angle, and at about 1.7 second, the torque is gradually released,the rudder and the motor return to the start point simultaneously.

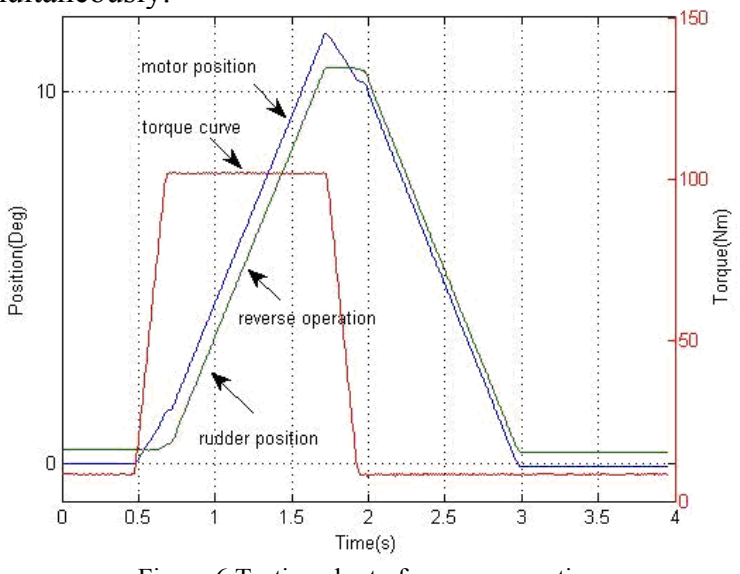

Figure.6 Testing chart of reverse operation

Fig. 7 shows the curve of reverse operation and positive operation torque load on rudder and the coordinate in Fig.8 has the same meaning with that of Fig.6. At the reverse operation stage, a $50 \mathrm{Nm}$ torque is imposed on the rudder and the motor rotates $10^{\circ}$, and after positive operation stage, the rudder and the motor return to the start point simultaneously.

According to the above definition,torque direction imposed on the rudder shaft by loading motor in a positive operation stage is contrary with the rudder motion direction. In Fig.7, position difference meets the relationship in (3).

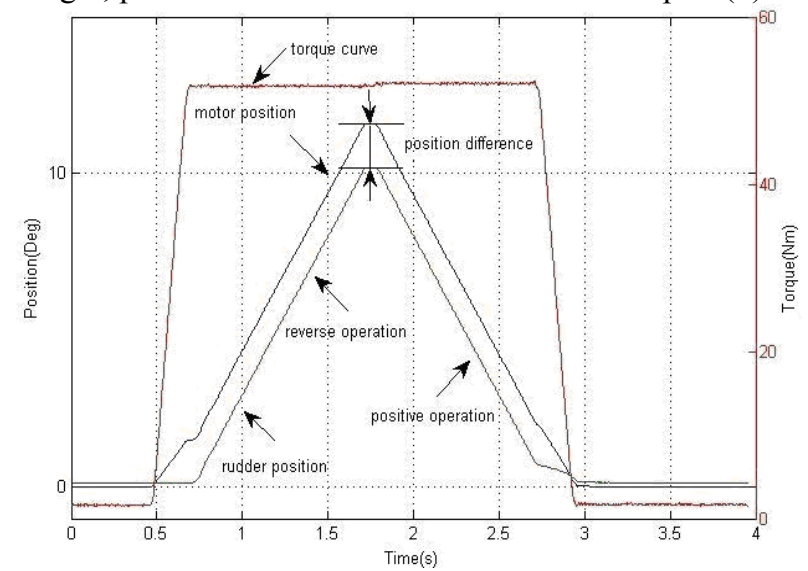

Figure. 7 Testing chart of positive and reverse operation

\section{CONCLUSIONS}

To meet the testing requirement of reverse operation load for new rudders, the large torque electrical load for positive and reverse operation is designed. The simulator is convenient to realize not only the torque load for positive and reverse operation but also the load testing for other signal, such as sinusoidal and square wave signal.

The system works well for positive and reverse operation of rudder and has a compact structure, and high precision, high symmetry and high linearity of load torque. Meanwhile, the spring sticks with different stiffness are designed according to different rudder and proper control parameters can be chosen to obtain best torque loading performance. The actual testing results show that load frequency can exceed $20 \mathrm{~Hz}$ under certain rudder angle and certain torque amplitude, and it can also meet "double five" and "double ten" index. The loading equipment has been delivered to the customer. The goal of anticipated design is achieved, and the results are satisfying.

\section{REFERENCES}

[1] Cao Tong, Sun Xingchu, Ouyang Qin, et al, "Design of loading stand for reverse operation of navigating machine," Journal of Beijing University of Aeronautics and Astronautics,Vol.29,No.2,March 2003, pp.252-254.

[2] Wang Mingyan, "Research on electrical powered load simulation," Harbin Institute of Technology,Ph.D. thesis,2004,pp.16-20.

[3] Fan Jinhua, "Research on aerobomb actuator loading system,"National university of defense tchnology, Master's thesis,2007,pp.8-15.

[4] Xin Wang,Dongzhu Feng, Haoyu Hu,"Design and Experiment of Rudder Load Simulator for High Dynamic Aircraft," Proceeding of the ICIEA,2009,pp.2573-2577.

[5] Fu Wenxing, Zhu Supeng, Sun Li, "Research of spring bar stiffness effect on performance of electric load simulator," Journal of 
Projectiles, Rockets, Missiles and Guidance, Vol.29,No.2,April 2009,pp.286-288.

[6] Wang Weijie,Li Donghai,Gao Qirui,et.al,“ Two-degrees-of-freedom PID controller tuning method,"Journal of Tsinghua University(Science and Technology),Vol.48,No.11,Nov. 2008,pp. 1962-1966.
[7] Xin Wang,Dongzhu Feng,"A study on dynamics of electric load simulator using spring beam and feedforward control technique,'IEEE Chinese Control and Decision Conference,2009, pp.301-306. 